\title{
Cancer-specific variation in emergency presentation by sex, age and deprivation across 27 common and rarer cancers
}

\author{
G A Abel ${ }^{\star, 1}$, J Shelton ${ }^{2,3}$, S Johnson ${ }^{3}$, L Elliss-Brookes ${ }^{3}$ and G Lyratzopoulos ${ }^{1,3,4}$ \\ ${ }^{1}$ Cambridge Centre for Health Services Research, Department of Public Health and Primary Care, Institute of Public Health, Forvie \\ Site, Robinson Way, Cambridge CB2 OSR, UK; ${ }^{2}$ Care Quality Commission, Finsbury Tower, 103-105 Bunhill Row, London EC1Y \\ 8TG, UK; ${ }^{3}$ National Cancer Intelligence Network (NCIN), Public Health England, 5th Floor, Wellington House, 135-155 Waterloo \\ Road, London SE1 8UG, UK and ${ }^{4}$ Health Behaviour Research Centre, Department of Epidemiology and Public Health, University \\ College London, 1-19 Torrington Place, London WC1E 6BT, UK
}

Background: Although overall sociodemographic and cancer site variation in the risk of cancer diagnosis through emergency presentation has been previously described, relatively little is known about how this risk may vary differentially by sex, age and deprivation group between patients with a given cancer.

Methods: Data from the Routes to Diagnosis project on 749645 patients (2006-2010) with any of 27 cancers that can occur in either sex were analysed. Crude proportions and crude and adjusted odds ratios were calculated for emergency presentation, and interactions between sex, age and deprivation with cancer were examined.

Results: The overall proportion of patients diagnosed through emergency presentation varied greatly by cancer. Compared with men, women were at greater risk for emergency presentation for bladder, brain, rectal, liver, stomach, colon and lung cancer (e.g., bladder cancer-specific odds ratio for women vs men, 1.50; $95 \% \mathrm{Cl} 1.39-1.60)$, whereas the opposite was true for oral/ oropharyngeal cancer, lymphomas and melanoma (e.g., oropharyngeal cancer-specific odds ratio for women vs men, $0.49 ; 95 \% \mathrm{Cl}$ 0.32-0.73). Similarly, younger patients were at higher risk for emergency presentation for acute leukaemia, colon, stomach and oesophageal cancer (e.g., colon cancer-specific odds ratio in 35-44- vs 65-74-year-olds, 2.01; 95\% Cl 1.76-2.30) and older patients for laryngeal, melanoma, thyroid, oral and Hodgkin's lymphoma (e.g., melanoma specific odds ratio in 35-44- vs 65-74-year-olds, $0.20 ; 95 \% \mathrm{Cl} 0.12-0.33)$. Inequalities in the risk of emergency presentation by deprivation group were greatest for oral/ oropharyngeal, anal, laryngeal and small intestine cancers.

Conclusions: Among patients with the same cancer, the risk for emergency presentation varies notably by sex, age and deprivation group. The findings suggest that, beyond tumour biology, diagnosis through an emergency route may be associated both with psychosocial processes, which can delay seeking of medical help, and with difficulties in suspecting the diagnosis of cancer after presentation.

A substantial proportion of cancer patients are diagnosed through an emergency route (hereafter described as emergency presentation) (Elliss-Brookes et al, 2012; Gunnarsson et al, 2013). Reducing this proportion is desirable for at least three reasons. First, diagnosis of cancer through an emergency presentation is associated with poorer survival outcomes (Sjo et al, 2009;
Elliss-Brookes et al, 2012; McPhail et al, 2013). Second, although being diagnosed with cancer is typically a stressful life event in itself, diagnosis through an emergency presentation increases stress, anxiety and inconvenience for patients and their relatives. Third, because patients diagnosed through emergency presentations typically require urgent management, pressure on the out-of-hours capacity of the

*Correspondence: Dr GA Abel; E-mail: ga302@medschl.cam.ac.uk

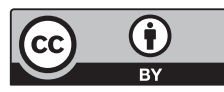


health-care system is intensified, and may itself contribute to poorer outcomes (Iversen et al, 2008; McPhail et al, 2013).

Despite a justifiable desire to reduce emergency presentations, there is uncertainty about how this can be achieved. Some emergency presentations may reflect purely biological factors: tumour type, aggressiveness or anatomical location may lead to sudden and critical clinical presentations with minimal or no prodromal symptoms (and, therefore, no opportunity for prior contact with the formal health-care system). In such circumstances, emergency presentations can be deemed unavoidable (Lyratzopoulos et al, 2014). However, in other patients, symptoms would have preceded the emergency presentation, but patients themselves or their doctors may not have promptly appreciated their likely importance (Lyratzopoulos et al, 2014). Therefore, diagnoses through emergency presentation may, in addition to tumour biology, reflect psychosocial factors preventing prompt presentation and help-seeking (Robb et al, 2009; Beeken et al, 2011), or diagnostic difficulty by doctors in the presence of atypical symptoms (Hamilton, 2012; Sheringham et al, 2014).

Although the exact contribution of biological, patient and healthcare factors is unknown, studies have documented that many patients diagnosed with cancer through an emergency presentation have had prior general practitioner consultations (Barrett and Hamilton, 2008), often for atypical symptoms (Cleary et al, 2007; Sheringham et al, 2014), but some have had no prior contact with primary care. Therefore, potential reductions in the frequency of emergency presentations may be achievable both by improving the effectiveness of the diagnostic process during primary care encounters and by reducing the proportion of patients who are diagnosed as emergencies without prior help-seeking.

Insights into potential responsible mechanisms may be obtained by appreciating variation in the risk of emergency presentation. Large variations in this risk by cancer diagnosis, age and socioeconomic status have been previously reported (EllissBrookes et al, 2012; National Cancer Intelligence Network, 2014). However, for patients with a given cancer, the risk of emergency presentation may differ by patient characteristic, and variably so for different cancers. Appreciating such variation may further help identify the mechanisms contributing to these risks, which can then be targeted by interventions. Against this background we aimed to examine cancer-specific variation in emergency presentation by sex, deprivation group and age among patients with common and rarer cancers that can occur in either sex.

\section{MATERIALS AND METHODS}

Data. Data were extracted from the National Cancer Data repository for England. The methods used to assign the route to diagnosis have been described in detail previously (Elliss-Brookes et al, 2012; National Cancer Intelligence Network, 2014). Briefly, all patients diagnosed with cancer during the study period (20062010) were assigned to a diagnostic 'route'. 'Routes' can be conceptualised as different pathways to diagnosis. They included emergency presentation and other routes, such as primary care referral for suspected cancer (either a '2-week wait' or routine referral) and screening.

Using cancer registration records as the base data set, additional information from Hospital Episodes Statistics, National Cancer Waiting Times and National Health Service screening programmes data (for breast, bowel and cervical cancers) were linked at persontumour level. The emergency presentation route denotes a new diagnosis of cancer after an emergency hospital admission or Accident and Emergency department attendance, or after emergency hospital transfer or emergency general practitioner referral; detailed operational definitions and algorithms have been further stipulated in detail (Elliss-Brookes et al, 2012; National Cancer Intelligence Network, 2014).

For the present study, data relate to patients aged 25 years or older with one of 27 non-sex-specific malignancies, aiming for the largest possible number of cancers that can occur in either sex with an adequate sample size - that is, anal, bladder, brain, colon, Hodgkin's lymphoma, laryngeal, liver, lung, melanoma, mesothelioma, multiple myeloma, non-Hodgkin's lymphoma, thyroid, oesophageal, oral, oropharyngeal, renal, rectal, pancreatic, small intestine, soft-tissue sarcoma, stomach and cancer of unknown primary, in addition to four leukaemia groups (acute lymphoblastic leukaemia (ALL), acute myeloid leukaemia (AML), chronic lymphocytic leukaemia (CLL) and chronic myeloid leukaemia $(\mathrm{CML})$ ). Diagnostic code definitions as per the International Classification of Diseases-10 for each diagnostic group are provided in Supplementary Online Material 1. Information was also available for age at diagnosis, sex, year of diagnosis and areabased deprivation of patients' home address (Index of Multiple Deprivation 2010, income domain only, Department for Communities and Local Government, 2011). Ages were aggregated into 10-year age groups, apart from those over 85 years, which were treated as a single group (after excluding those aged over 99 following the study by Elliss-Brookes et al, 2012). Deprivation was split into five groups on the basis of national quintiles.

Analysis. We first described the crude proportion of patients who were diagnosed through emergency presentation by variable category (i.e., by age group, sex, deprivation group, year of diagnosis and cancer diagnosis) and calculated the respective crude odds ratios. Adjusted odds ratios were estimated using a logistic regression model that, conditional on being a cancer case, predicts the emergency presentation status (yes/no binary variable) adjusting for age, sex, deprivation group, year of diagnosis and cancer (model 1).

Subsequently, we examined whether the effect of sex, deprivation and age varied between cancers by including two-way interactions between cancer and each of the three sociodemographic characteristics in the above model. Each of the three interaction terms was tested in turn with a single joint test. Statistically significant interaction terms $(P<0.05)$ were retained in the model (model 2$)$, which was then used to estimate cancer-specific odds ratios for emergency presentation, by sex, age and deprivation. When all three interaction terms are retained, this model is almost equivalent to a series of models stratified by cancer (except for the shared effect of year across cancers). This model was also used to predict the risk of emergency presentation for all-cancer-age-sex-deprivation group strata for cases diagnosed in 2010.

In both multivariable models the presence of overdispersion was assessed by the Pearson $\chi^{2}$-statistic and by examination of the distribution of deviance residuals. Overdispersion was then accounted for by scaling the standard errors appropriately. All analyses were carried out in Stata v13.1 (StataCorp, College Station, TX, USA).

\section{RESULTS}

There were 749645 incident tumours contained within the 27 nonsex-specific cancer diagnosis groups under consideration; among those the diagnosis was through an emergency presentation in 232281 (31\%) cases (Table 1). This percentage is higher than previously published 'all-cancer' averages (Elliss-Brookes et al, 2012; National Cancer Intelligence Network, 2014) primarily because of the exclusion of the sex-specific sites (including prostate and breast cancers, two common cancers with low proportions of emergency presentations).

Overall variation in emergency presentation by age, sex, deprivation, diagnosis year and cancer (model 1 findings). There was strong evidence for variation in emergency presentation 
Table 1. Crude proportion, and crude and adjusted odds ratios for diagnosis through emergency presentation, by age group, sex, deprivation group, year of diagnosis and cancer diagnosis

\begin{tabular}{|c|c|c|c|c|c|}
\hline & & Emerge & tation & Odds $r$ & $5 \%(\mathrm{Cl})^{a}$ \\
\hline & All cases & Number & $\%$ & Crude & Adjusted \\
\hline All Cases & 749645 & 232281 & $31.0 \%$ & N/A & N/A \\
\hline Age & & & & & \\
\hline $25-34$ & 8586 & 1659 & $19.3 \%$ & $0.65(0.62-0.69)$ & $1.10(1.00-1.21)$ \\
\hline $35-44$ & 22356 & 4481 & $20.0 \%$ & $0.68(0.66-0.70)$ & $1.01(0.95-1.07)$ \\
\hline $45-54$ & 57128 & 12920 & $22.6 \%$ & $0.79(0.78-0.81)$ & $0.94(0.90-0.97)$ \\
\hline $55-64$ & 140074 & 33741 & $24.1 \%$ & $0.86(0.85-0.87)$ & $0.91(0.89-0.93)$ \\
\hline $65-74$ & 208095 & 56025 & $26.9 \%$ & Reference & Reference \\
\hline $75-84$ & 218951 & 77723 & $35.5 \%$ & $1.49(1.47-1.51)$ & $1.47(1.45-1.50)$ \\
\hline $85+$ & 94455 & 45732 & $48.4 \%$ & $2.55(2.51-2.59)$ & $2.62(2.56-2.69)$ \\
\hline Sex & & & & & \\
\hline Men & 428155 & 126300 & $29.5 \%$ & Reference & Reference \\
\hline Women & 321490 & 105981 & $33.0 \%$ & $1.18(1.16-1.19)$ & $1.06(1.04-1.08)$ \\
\hline Deprivation group & & & & & \\
\hline 1-Least deprived & 138269 & 36255 & $26.2 \%$ & Reference & Reference \\
\hline 2 & 155743 & 44332 & $28.5 \%$ & $1.12(1.10-1.14)$ & $1.09(1.06-1.11)$ \\
\hline 3 & 157940 & 48256 & $30.6 \%$ & $1.24(1.22-1.26)$ & $1.19(1.16-1.22)$ \\
\hline 4 & 152953 & 50779 & $33.2 \%$ & $1.40(1.38-1.42)$ & $1.33(1.30-1.36)$ \\
\hline 5-Most deprived & 144740 & 52659 & $36.4 \%$ & $1.61(1.58-1.64)$ & $1.55(1.51-1.59)$ \\
\hline Year of diagnosis & & & & & \\
\hline 2006 & 146259 & 47987 & $32.8 \%$ & Reference & Reference \\
\hline 2007 & 147473 & 46333 & $31.4 \%$ & $0.94(0.92-0.95)$ & $0.93(0.91-0.96)$ \\
\hline 2008 & 152125 & 46784 & $30.8 \%$ & $0.91(0.90-0.92)$ & $0.91(0.89-0.93)$ \\
\hline 2009 & 153600 & 46890 & $30.5 \%$ & $0.90(0.89-0.91)$ & $0.90(0.88-0.92)$ \\
\hline 2010 & 150188 & 44287 & $29.5 \%$ & $0.86(0.84-0.87)$ & $0.87(0.85-0.89)$ \\
\hline Cancer diagnosis & & & & & \\
\hline Melanoma $^{b}$ & 45561 & 967 & $2.1 \%$ & $0.03(0.03-0.04)$ & $0.04(0.04-0.04)$ \\
\hline Oral & 9801 & 491 & $5.0 \%$ & $0.08(0.08-0.09)$ & $0.09(0.08-0.10)$ \\
\hline Thyroid & 8254 & 460 & $5.6 \%$ & 0.09 (0.09-0.10) & $0.11(0.10-0.13)$ \\
\hline Oropharynx & 6429 & 365 & $5.7 \%$ & $0.10(0.09-0.11)$ & $0.12(0.10-0.14)$ \\
\hline Anal & 3381 & 345 & $10.2 \%$ & $0.18(0.16-0.20)$ & $0.19(0.16-0.22)$ \\
\hline Laryngeal & 8283 & 833 & $10.1 \%$ & $0.18(0.17-0.19)$ & $0.20(0.18-0.22)$ \\
\hline Soft tissue sarcoma & 4839 & 635 & $13.1 \%$ & $0.24(0.22-0.26)$ & $0.26(0.23-0.29)$ \\
\hline Rectal & 54076 & 8177 & $15.1 \%$ & $0.29(0.28-0.29)$ & $0.30(0.28-0.31)$ \\
\hline Hodgkin lymphoma & 4768 & 674 & $14.1 \%$ & $0.26(0.24-0.29)$ & $0.31(0.28-0.35)$ \\
\hline Bladder $^{\mathbf{b}}$ & 42234 & 7834 & $18.5 \%$ & $0.36(0.36-0.37)$ & $0.34(0.33-0.35)$ \\
\hline Oesophageal & 32470 & 7062 & $21.7 \%$ & $0.44(0.43-0.46)$ & $0.45(0.43-0.47)$ \\
\hline & 11892 & 2950 & $24.8 \%$ & $0.53(0.51-0.55)$ & $0.53(0.49-0.56)$ \\
\hline Renal $^{\mathbf{b}}$ & 29469 & 7733 & $26.2 \%$ & $0.57(0.55-0.59)$ & $0.62(0.60-0.65)$ \\
\hline Non-Hodgkin ${ }^{b}$ lymphoma & 46329 & 12393 & $26.7 \%$ & $0.58(0.57-0.60)$ & $0.64(0.62-0.66)$ \\
\hline Colon & 97880 & 30777 & $31.4 \%$ & $0.73(0.72-0.75)$ & $0.73(0.71-0.75)$ \\
\hline Stomach $^{\mathbf{b}}$ & 29893 & 9913 & $33.2 \%$ & $0.79(0.77-0.82)$ & $0.75(0.73-0.78)$ \\
\hline Multiple myeloma ${ }^{\mathbf{b}}$ & 18272 & 6693 & $36.6 \%$ & $0.93(0.90-0.96)$ & $0.96(0.91-1.00)$ \\
\hline Mesothelioma & 10116 & 3631 & $35.9 \%$ & $0.90(0.86-0.93)$ & $0.96(0.90-1.02)$ \\
\hline Lung $^{b}$ & 162543 & 62498 & $38.5 \%$ & Reference & Reference \\
\hline $\mathrm{CML}$ & 1702 & 656 & $38.5 \%$ & $1.00(0.91-1.11)$ & $1.01(0.88-1.17)$ \\
\hline Pancreatic $^{\mathbf{b}}$ & 33295 & 16364 & $49.1 \%$ & $1.55(1.51-1.58)$ & $1.56(1.51-1.62)$ \\
\hline Liver & 14732 & 7270 & $49.3 \%$ & $1.56(1.51-1.61)$ & $1.60(1.52-1.68)$ \\
\hline Unknown primary & 43290 & 24805 & $57.3 \%$ & $2.15(2.10-2.19)$ & 2.00 (1.94-2.07) \\
\hline AML & 9611 & 5388 & $56.1 \%$ & $2.04(1.96-2.13)$ & $2.15(2.02-2.29)$ \\
\hline Small intestine & 3399 & 1863 & $54.8 \%$ & $1.94(1.81-2.08)$ & $2.15(1.95-2.38)$ \\
\hline Brain $^{b}$ & 16710 & 11175 & $66.9 \%$ & $3.23(3.12-3.34)$ & $3.96(3.77-4.17)$ \\
\hline ALL & 416 & 329 & $79.1 \%$ & 6.05 (4.78-7.67) & $7.19(5.08-10.16)$ \\
\hline $\begin{array}{l}\text { Abbreviations: } A L L=\text { acute lyn } \\
N A=\text { not applicable. } \\
{ }^{\mathbf{P}_{P}} P<0.0001 \text { for all based on joi } \\
\mathbf{b}_{\text {See also Elliss-Brookes et al, }}\end{array}$ & $\begin{array}{l}\text { a; } \mathrm{AML}=\mathrm{acu} \\
\text { variables. } \mathrm{A}\end{array}$ & $\begin{array}{l}\text { raemia; } \mathrm{Cl}= \\
\text { of overdisp }\end{array}$ & erval; $\mathrm{CL}$ & $\begin{array}{l}\text { hocytic leukaemia; } \\
\text { in the width of cor }\end{array}$ & $\begin{array}{l}\text { hronic myeloid leuka } \\
\text { ntervals of } 45 \% \text {. }\end{array}$ \\
\hline
\end{tabular}

by sex, deprivation group, age, year of diagnosis and cancer diagnosis $(P<0.0001$ for all; Table 1$)$. Specifically, there was a small excess risk of emergency presentation among women compared with men (adjusted odds ratio, 1.06; 95\% CI 1.04-1.08). The risk of emergency presentation increased with each increasing deprivation group (adjusted odds ratio for most $v s$ least deprived group, 1.55; 95\% CI 1.51-1.59). Crudely, the risk of emergency diagnosis increased with age across all ages considered. However, after adjusting for other variables there was a slight increase in risk with decreasing age for those under 55 years, but those most at risk were still the older patients (e.g., adjusted odds ratio for ages 75-84 vs 6574 years, 1.47 ; $95 \%$ CI 1.45-1.50).

The crude risk of emergency presentation decreased year-on-year, with diagnosis year being highest (33\%) at the baseline year (2006) 
and lowest (30\%) in the last year (2010) of the study period $(P<0.0001)$. A consistent pattern was seen in the adjusted findings.

There was very large variation in the crude risk for emergency presentation by cancer, being lowest for melanoma and oral cancer ( $2 \%$ and $5 \%$, respectively) and very high for patients with brain cancer and ALL (67\% and 79\%, respectively; Table 1). This large variation persisted after adjustment for other variables, with about a 180-fold variation in the adjusted odds of emergency presentation across cancer diagnoses (i.e., odds ratio comparing ALL to melanoma $7.19 / 0.04=179.31)$.

Cancer-specific variation in emergency presentation by age, sex and deprivation (model 2 findings). There was very strong evidence $(P<0.0001$ for each of the three two-way interaction terms) that the effect of sex, age and deprivation varied between the studied cancers. Figure 1 shows cancer-specific sex, age (35-44 vs 65-74 years) and deprivation (most $v s$ least deprived) associations. Note that, although a modest amount of overdispersion was present (resulting in an increase in the width of confidence intervals of $31 \%$ ), the distribution of deviance residuals was very close to normal. Variability between cancers is clearly largest for the effect of age; furthermore, the largest age effects tend to be larger than either the sex or deprivation effects. We discuss each of the sociodemographic variables in detail below.

Sex. There was evidence $(P<0.05)$ that women had greater risk of emergency presentation than men for seven cancers - namely, in descending order of effect size, bladder, brain, rectal, liver, stomach, colon and lung cancer (odds ratios for women vs men ranging from 1.50 to 1.05 for bladder and lung cancer, respectively; Figure 1A). Conversely, there was evidence $(P<0.05)$ that women had lower risk of emergency presentation compared with men for five cancers - namely, in descending order of effect size, oropharyngeal, oral, Hodgkin's lymphoma, melanoma and nonHodgkin's lymphoma (odds ratios for women $v s$ men ranging from 0.49 to 0.88 for oropharyngeal and non-Hodgkin's lymphoma, respectively). There was no evidence for variation by sex in the risk of emergency presentation for the other 12 cancers examined; for some of those cancers this may reflect the small number of patients concerned.

Deprivation. There was evidence $(P<0.05)$ that patients in the most deprived groups were at greater risk of emergency presentation in nearly all (24 out of 27) cancers (Figure 1B). Within this overall consistent pattern, excess risk of emergency presentation among more deprived patients was particularly pronounced for patients with five cancers - namely, oral, oropharyngeal, anal, small intestine and laryngeal cancers (odds ratios (most $v s$ least deprived patients) ranging from 3.82 to 2.18 for patients with oral and laryngeal cancer, respectively). The three cancers without evidence for variation by deprivation were ALL, CML and soft-tissue sarcoma - the first of which had very few cases and hence very large uncertainty around the estimate.

Age. Although on average older patients had higher risk of emergency presentation, the presence, size and direction of associations with age varied notably between patients with different cancers. The comparison of 35-44-year-olds with 65-74-year-olds is useful as a direct comparison with sex and deprivation effects (Figure 1C), but for a full understanding of the cancer-specific age patterns variation across all age groups ought to be considered (Figure 2). Three patterns can be initially distinguished. For some cancers, the risk of emergency presentation increases with age across all age groups (laryngeal, melanoma, thyroid, oral, anal, brain, oropharynx, renal cancer and soft-tissue sarcoma). For one cancer (ALL), the reverse pattern was observed. For the remaining cancers (AML, colon, stomach, oesophageal, liver, bladder,
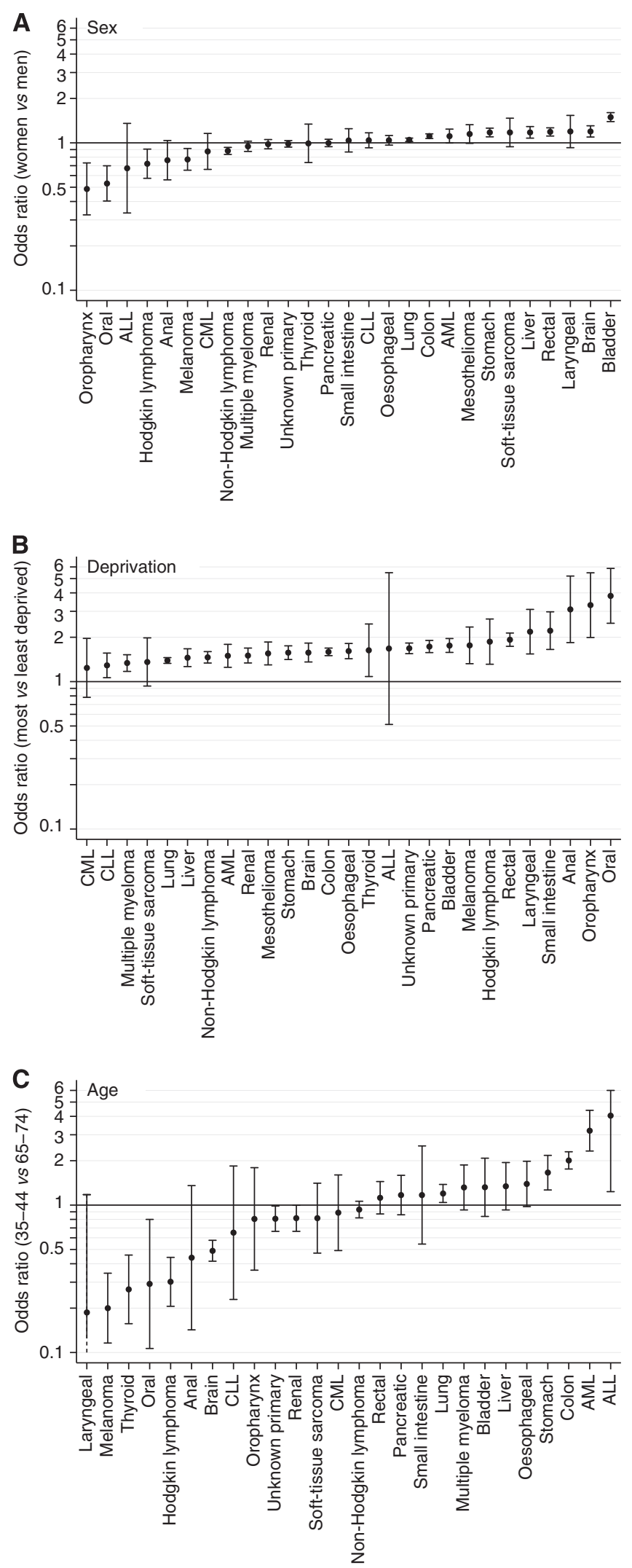

Figure 1. Cancer-specific odds ratios and $95 \%$ confidence intervals for emergency presentation comparing (A) women vs men; (B) most vs least deprived patients; and (C) 35-44-year-olds vs 65-74-year-olds. Note that mesothelioma is not displayed in C, see Figure 2 for details. 


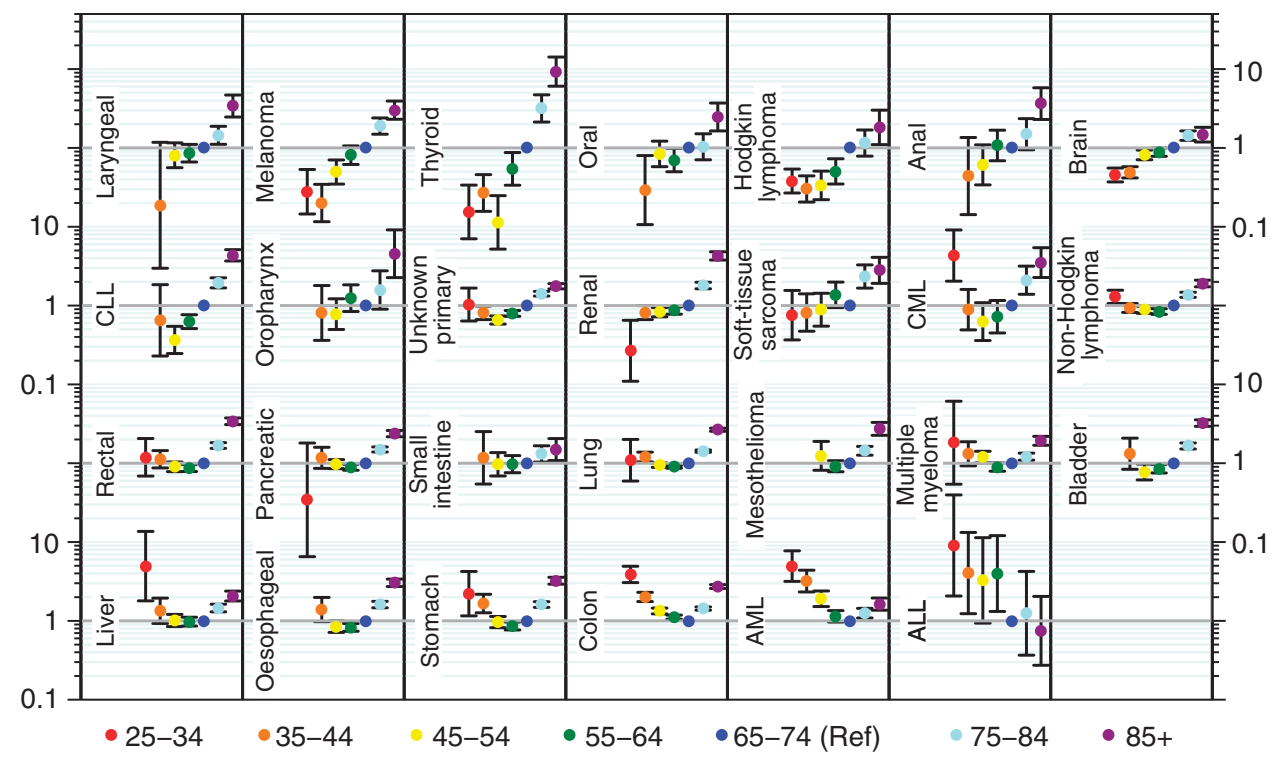

Figure 2. Cancer-specific odds ratios and $95 \%$ confidence intervals for emergency presentation by age group compared with $65-74$ years (reference). Note that where cancer-specific age groups contained no cases or all cases were either emergency or non-emergency presentations, odds ratios cannot be estimated and are not shown. This relates to the two younger age groups (25-34 or 35-44), for a total of 30 individual tumours across nine cancers.

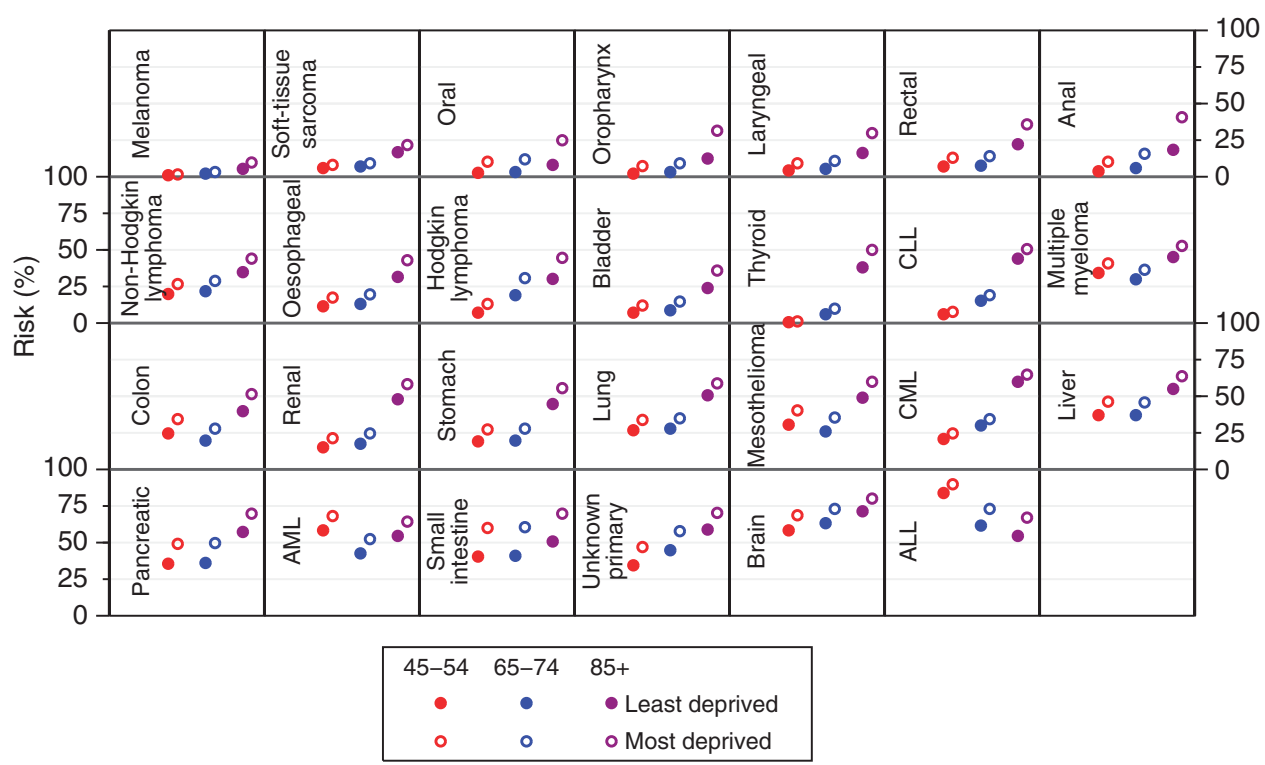

Figure 3. Predicted risk of diagnosis of cancer through an emergency presentation for men in three age groups (45-54, $65-74$ and $85+$ years), two deprivation groups (most and least deprived) and with 27 cancers. Confidence intervals can be found in Supplementary Online Material 2.

multiple myeloma, mesothelioma, lung cancer, small intestine, pancreatic, rectal, non-Hodgkin's lymphoma, CLL, CML and cancer of unknown primary), either a J- or a U-shaped relationship with age was apparent, such that the risk of emergency presentation initially decreased and then increased with age, with the oldest patients in most cases being those most at risk. There was wide variability in the overall size of age effects, which were particularly large for cancers such as thyroid, melanoma and ALL, decreasing to almost no age effect for small intestine cancer.

The appreciable size of cancer-specific sociodemographic variation in the risk of emergency presentation is further illustrated by the predicted risk of emergency presentations. This is shown in full for each cancer-age-sex-deprivation group strata for cases diagnosed in 2010 in Supplementary Online Material 2. We also show the predicted risk for men for three age groups (45-54, 65-74 and 85-99) and two deprivation groups (least and most deprived; Figure 3). In particular, we note that the sociodemographic variation introduces considerable within-cancer variation. For example, for thyroid, renal, pancreatic and ALL, we see that the risk of diagnosis through an emergency presentation between extreme the age group-deprivation strata shown in Figure 3 varies between $1 \%$ and $50 \%, 15 \%$ and $58 \%, 36 \%$ and $70 \%$ and $55 \%$ and $90 \%$, respectively.

\section{DISCUSSION}

We describe notable cancer-specific differences in the risk of emergency presentation by patient characteristic among patients 
with 27 common and rarer cancers that can occur in either sex. Despite the study period being relatively short (i.e. 5 years), we also observed a decreasing trend in emergency presentations over time.

Comparisons with previous evidence (what is known about the subject and what does this study add). Although previous work has addressed variation in the risk of emergency presentation by cancer site and sociodemographic characteristics, our study examines how this risk varies for patients with a given cancer but with different sex, age and deprivation status.

Regarding overall patterns of variation in the risk of emergency presentation by cancer, with few differences in diagnostic group definitions, the findings iterate previous evidence encompassing between 15 and 56 cancers (Elliss-Brookes et al, 2012; National Cancer Intelligence Network, 2014). However, in this study we have demonstrated that variation by cancer persists after adjusting for age, sex and deprivation. Relatedly, it should be noted that 'linear' crude patterns of decreasing risk for emergency presentation in younger age groups disappear after case-mix adjustment (Table 1). This is chiefly a demonstration of the differential age case-mix of various cancer sites with, for example, melanoma (the site with the lowest proportion of emergency presentations) being relatively common in younger age groups (28\% of all $25-44$-yearolds with non-sex-specific cancers included in this study). Similarly, the higher odds of emergency presentation in women were substantially attenuated after case-mix adjustment; in contrast, there was little apparent confounding of deprivation differences in risk of emergency presentation by other variables (Table 1). The present study, additionally, describes time trends (year-on-year) more clearly and considers colon and rectal cancer separately for the first time, indicating that the proportion of emergency presentation is two-fold greater for patients with colon compared with rectal cancer. Symptom signatures of rectal and colon cancer are likely to vary - as also shown for other colorectal cancer subsites (Hamilton, 2005). Another study also indicates that colon cancer is associated with substantially higher proportion of multiple pre-referral consultations compared with rectal cancer after presentation in primary care (Lyratzopoulos et al, 2012).

Regarding sociodemographic predictors of emergency presentation, the findings reiterate previously reported patterns of variation both from the Routes to Diagnosis project and from other settings, indicating higher risk of emergency presentation among older patients, those with lower socioeconomic status, and among women (Rabeneck et al, 2006; Bottle et al, 2012; Elliss-Brookes et al, 2012; National Cancer Intelligence Network, 2014; Wallace et al, 2014). However, this study also demonstrates that the presence, size and direction of sociodemographic differences in emergency presentation vary considerably between patients with a given cancer.

Strengths and limitations. Our study uses population-based data of high quality for many different cancers and a relatively recent study period, and validated methodologies to define and assign the diagnosis of cancer through an emergency presentation. Sample attrition due to missing sociodemographic or cancer diagnosis information was trivial, given the very high completeness of this information in the primary sources used in the Routes to Diagnosis project. A small but non-trivial proportion of cases (4\%) have been assigned an 'unknown' route, as information was not available about the diagnosis route for these tumours from routine data sets. These have been treated as 'non-emergency presentations' for the purposes of this analysis, as with our operational definition it is impossible to have an emergency presentation without having a signal in Hospital Episode Statistics. There was no information on symptom at presentation, nor about the exact circumstances preceding emergency presentation - more specifically, whether the presentation was the first ever contact with the health-care system, or whether patients had previously consulted for symptoms either in primary or in secondary care.

Interpretation and implications. The Routes to Diagnosis project reveals a wealth of evidence that allows for a highly refined and stratified understanding of the risk of emergency presentation, not only by sociodemographic characteristics and cancer but also by their interactions. Given the large size and multiple types of variation revealed, it would be imprudent to attempt to 'explain' all observed findings where notable variation was detected. Instead we propose that appreciation of the large and complex nature of the variations we describe should motivate future clinical, epidemiological and behavioural science research. We nonetheless provide a few hypotheses about some aspects of the findings. Two observations can help to inform the consideration of the findings.

First, most known sociodemographic inequalities in markers of diagnostic timeliness (such as stage at diagnosis) are concentrated on cancers with relatively clear-cut symptom signatures-that is cancers in which the majority of patients present with visible, palpable or audible symptoms (Lyratzopoulos and Abel, 2013; Lyratzopoulos et al, 2013a; Robbins et al, 2014). These include melanoma, oral/oropharyngeal, anal and laryngeal cancers (and also endometrial, breast and testicular cancer, not examined here as sex-specific cancers). Bearing this observation in mind it is apparent that, where present, cancer-specific inequalities (by sex, deprivation and age) tend to relate to cancers characterised by clear-cut symptoms in most patients (i.e., oral/oropharygeal cancer oral ulceration/lesion; melanoma - visible skin lesion; anal - anal ulceration/lesions; and laryngeal - voice hoarseness). For example, cancer-specific variation in risk of emergency presentation by deprivation group was particularly pronounced for oral, oropharyngeal and laryngeal cancers. These patterns would seem to indicate a reduced ability or motivation by poorer patients to appropriately appraise and seek medical help (Walter et al, 2012) for easily noticeable bodily changes compared with more affluent patients, in turn prolonging the patient interval and increasing the risk of emergency presentation (Lyratzopoulos et al, 2014). Variation in the risk of emergency presentation by deprivation was, among others, pronounced for anal and rectal cancer, which might suggest a degree of socioeconomic patterning of stigma or embarrassment associated with gastrointestinal symptoms.

Second, although emergency presentations are multifactorial, they do seem to represent a marker of 'diagnostic difficulty' after presentation. This becomes apparent when appreciating that 'harder-to-suspect' cancers (i.e., those associated with the highest proportions of multiple pre-referral primary care consultations, such as multiple myeloma, stomach, pancreatic and colon cancer) also have a high proportion of emergency presentations (Table 1) (Hamilton, 2012; Lyratzopoulos et al, 2012, 2013b). Conversely, 'easier-to-suspect' cancers (such as melanoma and breast cancer) have the lowest proportion of emergency presentations (EllissBrookes et al, 2012; National Cancer Intelligence Network, 2014). Taking these observations into account, it can be noted that bladder cancer is associated with notable sex differences in the promptness of diagnosis after presentation, betraying a higher level of difficulty by doctors in suspecting the diagnosis of bladder cancer in symptomatic women (Lyratzopoulos et al, 2013c), and it is for bladder cancer that the highest excess risk of emergency presentation for women was also observed. It is therefore plausible that the excess risk of emergency diagnosis of women with bladder cancer reflects prolonged intervals to diagnosis after presentation.

Finally, there is a group of cancers for which men are at greater risk of diagnosis through an emergency presentation - for example, oral and oropharyngeal cancer (both characterised by clear-cut symptoms (oral ulceration/lesion) in most patients) and melanoma (visible skin lesions in most patients)). These observations would seem to suggest reduced levels of body consciousness and reduced 
ability to appraise and seek help for easily recognisable symptoms among men compared with women.

As remarked in the Results section, cancer-specific variation in risk of emergency presentation by age was much larger compared with variation by sex and deprivation. We would posit that, given its very large size, the greatest part of this variation reflects biological factors (i.e., differences in tumour type and aggressiveness between patients with cancers of the same site but in different age groups). However (bearing in mind that the excess risk in older patients was non-uniform between cancers but present in all but one; Figure 2), reduced help-seeking in older age may be another likely source, given lower levels of awareness of age-related risk of cancer and reduced knowledge about likely cancer symptoms in older individuals (Robb et al, 2009; Forbes et al, 2013). In addition, comorbidity (including mental health comorbidity, e.g., dementia syndromes) and/or social isolation/lack of support in older age may be contributing to excess risk of diagnosis through an emergency presentation - for the cancers in which such patterns are observed (Wallace et al, 2014). Conversely, younger patients had excess risk of emergency presentation for some cancers (e.g., CML, liver, colon, AML and ALL). Such instances may betray the greater difficulty in suspecting the diagnosis of these cancers in younger patients post presentation to a general practitioner (Lyratzopoulos et al, 2012), although why this pattern is apparent only for some cancers (Figure 2) should be the subject of further investigation.

We would like to emphasise that epidemiological data such as used in this study cannot determine the potential for avoiding the events of interest (emergency presentations), nor can they help accurately 'partition' the contribution of tumour, patient and healthcare factors in emergency presentation. Moreover, this study cannot determine which, or indeed what, proportion of emergency diagnosis may have been avoided, and how; further epidemiological research and retrospective case reviews are needed in this area (Lyratzopoulos et al, 2014). However, were all emergency diagnoses a manifestation of tumour factors, we would not be expecting to see the degree of cancer-specific variations by the sociodemographic variables that we have observed. Interventions should aim to reduce the proportion of emergency presentations by reducing the proportion that can be attributable to either health-care or patient factors.

The patterns of variation that we observe provide aetiological insights into factors other than tumour biology implicated in emergency presentations. They can therefore help to motivate and target the development and evaluation of interventions with a public health or health-care focus. We provide the research and policy-making community with ample evidence about the variable (stratified) risk of cancer diagnosis through an emergency presentation, which can be used to inform targeted interventions for different cancers/sociodemographic groups. As an example, among patients with oral or oropharyngeal cancer the risk of emergency presentation is higher for men, further increasing notably with increasing deprivation (Figure 1A and B). Future early diagnosis campaigns for cancers of the oral cavity can therefore focus on men from deprived communities. Cancers of the oral cavity are also known to be associated with the longest median patient interval compared to another 17 cancers (Keeble et al, 2014).

We also wish to note the particularly high proportion of patients with colon cancer who are diagnosed as emergencies (31\%)- twofold greater than for rectal cancer. An effective cancer control strategy for this common cancer is increasing the uptake of screening, which can be expected to decrease emergency presentations from colon cancer (Pruitt et al, 2014).

In conclusion, we have provided a comprehensive account of the descriptive epidemiology of cancer diagnosis through emergency presentation for patients with 27 different cancers, examining both overall and cancer-specific variation by patient characteristic. The findings suggest that, beyond tumour biology, emergency diagnosis may be associated with psychosocial processes delaying presentation and diagnostic difficulty post presentation; our findings also 'map out' the potential variable influence of patient (psychosocial), healthcare (diagnostic difficulty) and tumour-related factors. They can help motivate further research priorities and policy initiatives targeted at patient groups at greater risk of emergency presentation. Notable improvements over a short period of time have also been observed, indicating that to an extent the potential for decreasing emergency presentations is already being harvested.

\section{ACKNOWLEDGEMENTS}

We acknowledge the authors of previous studies that led to the creation and curation of the Routes to Diagnosis project and data set. The work presented here is a collaboration between Public Health England's National Cancer Intelligence Network and the Cambridge Centre for Health Services Research of the University of Cambridge. GL was supported by a post-doctoral fellowship by the National Institute for Health Research (PDF-2011-04-047) to the end of 2014 and by a Cancer Research UK Clinician Scientist Fellowship award (A18180) from 2015. The views expressed in this publication are those of the authors and not necessarily those of the NHS, the National Institute for Health Research, the Department of Health, the National Cancer Intelligence Network of Public Health England, Cancer Research UK, any other funding organisation.

\section{CONFLICT OF INTEREST}

The authors declare no conflict of interest.

\section{REFERENCES}

Barrett J, Hamilton W (2008) Pathways to the diagnosis of lung cancer in the UK: a cohort study. BMC Fam Pract 9: 31.

Beeken RJ, Simon AE, von Wagner C, Whitaker KL, Wardle J (2011) Cancer fatalism: deterring early presentation and increasing social inequalities? Cancer Epidemiol. Biomarkers Prev 20(10): 2127-2131.

Bottle A, Tsang C, Parsons C, Majeed A, Soljak M, Aylin P (2012) Association between patient and general practice characteristics and unplanned first-time admissions for cancer: observational study. Br J Cancer 107(8): 1213-1219.

Cleary J, Peters TJ, Sharp D, Hamilton W (2007) Clinical features of colorectal cancer before emergency presentation: a population-based case-control study. Fam Pract 24(1): 3-6.

Department for Communities and Local Government (2011) 'English indices of deprivation 2010.

Elliss-Brookes L, McPhail S, Ives A, Greenslade M, Shelton J, Hiom S, Richards M (2012) Routes to diagnosis for cancer-determining the patient journey using multiple routine data sets. Br J Cancer 107(8): $1220-1226$.

Forbes LJ, Simon AE, Warburton F, Boniface D, Brain KE, Dessaix A, Donnelly C, Haynes K, Hvidberg L, Lagerlund M, Lockwood G, Tishelman C, Vedsted P, Vigmostad MN, Ramirez AJ, Wardle J. International Cancer Benchmarking Partnership Module 2 Working Group (2013) Differences in cancer awareness and beliefs between Australia, Canada, Denmark, Norway, Sweden and the UK (the International Cancer Benchmarking Partnership): do they contribute to differences in cancer survival? Br J Cancer 108(2): 292-300.

Gunnarsson H, Ekholm A, Olsson LI (2013) Emergency presentation and socioeconomic status in colon cancer. Eur J Surg Oncol 39(8): 831-836.

Hamilton W (2012) Emergency admissions of cancer as a marker of diagnostic delay. Br J Cancer 107(8): 1205-1206.

Hamilton WT (2005) Towards earlier diagnosis of cancer in primary care: a population-based case-control study of colorectal, lung and prostate cancer. MD Thesis. University of Bristol: Bristol. 
Iversen LH, Bülow S, Christensen IJ, Laurberg S, Harling H. Danish Colorectal Cancer Group (2008) Postoperative medical complications are the main cause of early death after emergency surgery for colonic cancer. Br J Surg 95(8): 1012-1219.

Keeble S, Abel GA, Saunders CL, McPhail S, Walter FM, Neal RD, Rubin GP, Lyratzopoulos G (2014) Variation in promptness of presentation among 10,297 patients subsequently diagnosed with one of 18 cancers: evidence from a National Audit of Cancer Diagnosis in Primary Care. Int $J$ Cancer 135(5): 1220-1228.

Lyratzopoulos G, Abel GA (2013) Early diagnosis of breast cancer: focusing on symptomatic women. Nat Rev Clin Oncol 10(9).

Lyratzopoulos G, Abel GA, Brown CH, Rous BA, Vernon SA, Roland M, Greenberg DC (2013a) Socio-demographic inequalities in stage of cancer diagnosis: evidence from patients with female breast, lung, colon, rectal, prostate, renal, bladder, melanoma, ovarian and endometrial cancer. Ann Oncol 24(3): 843-850.

Lyratzopoulos G, Abel GA, McPhail S, Neal RD, Rubin GP (2013b) Measures of promptness of cancer diagnosis in primary care: secondary analysis of national audit data on patients with 18 common and rarer cancers. Br J Cancer 108(3): 686-690.

Lyratzopoulos G, Abel GA, McPhail S, Neal RD, Rubin GP (2013c) Gender inequalities in the promptness of diagnosis of bladder and renal cancer after symptomatic presentation: evidence from secondary analysis of an English primary care audit survey. BMJ Open 3(6): e002861.

Lyratzopoulos G, Neal RD, Barbiere JM, Rubin GP, Abel GA (2012) Variation in number of general practitioner consultations before hospital referral for cancer: findings from the 2010 National Cancer Patient Experience Survey in England. Lancet Oncol 13(4): 353-365.

Lyratzopoulos G, Saunders CL, Abel GA (2014) Are emergency diagnoses of cancer avoidable? A proposed taxonomy to motivate study design and support service improvement. Future Oncol 10(8): 1329-1333.

McPhail S, Elliss-Brookes L, Shelton J, Ives A, Greenslade M, Vernon S, Morris EJ, Richards M (2013) Emergency presentation of cancer and short-term mortality. Br J Cancer 109(8): 2027-2034.
National Cancer Intelligence Network (2014) Routes to Diagnosis http://www.ncin.org.uk/publications/routes_to_diagnosis.

Pruitt SL, Davidson NO, Gupta S, Yan Y, Schootman M (2014) Missed opportunities: racial and neighborhood socioeconomic disparities in emergency colorectal cancer diagnosis and surgery. BMC Cancer 14: 927.

Rabeneck L, Paszat LF, Li C (2006) Risk factors for obstruction, perforation, or emergency admission at presentation in patients with colorectal cancer: a population-based study. Am J Gastroenterol 101(5): 1098-1103.

Robb K, Stubbings S, Ramirez A, Macleod U, Austoker J, Waller J, Hiom S, Wardle J (2009) Public awareness of cancer in Britain: a population-based survey of adults. Br J Cancer 101(Suppl 2): S18-S23.

Robbins AS, Lerro CC, Barr RD (2014) Insurance status and distant-stage disease at diagnosis among adolescent and young adult patients with cancer aged 15 to 39 years: National Cancer Data Base, 2004 through 2010. Cancer 120(8): 1212-1219.

Sheringham JR, Georghiou T, Chitnis XA, Bardsley M (2014) Comparing primary and secondary health-care use between diagnostic routes before a colorectal cancer diagnosis: Cohort study using linked data. Br J Cancer 111(8): 1490-1499.

Sjo OH, Larsen S, Lunde OC, Nesbakken A (2009) Short term outcome after emergency and elective surgery for colon cancer. Colorectal Dis 11(7): 733-739.

Wallace D, Walker K, Kuryba A, Finan P, Scott N, van der Meulen J (2014) Identifying patients at risk of emergency admission for colorectal cancer. Br J Cancer 111(3): 577-580.

Walter F, Webster A, Scott S, Emery J (2012) The Andersen Model of Total Patient Delay: a systematic review of its application in cancer diagnosis. J Health Serv Res Policy 17(2): 110-118.

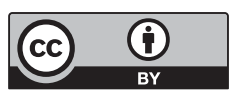

This work is licensed under the Creative Commons Attribution 4.0 International License. To view a copy of this license, visit http://creativecommons.org/licenses/by/4.0/

Supplementary Information accompanies this paper on British Journal of Cancer website (http://www.nature.com/bjc) 